\title{
Modelamiento estadístico y optimización del proceso de cianuración de un mineral aurífero
}

\author{
Jairo Antonio Ruiz-Córdoba ${ }^{1}$ \\ Carlos Alberto López-Cañas ${ }^{2}$ \\ María Eugenia Carmona-Arango ${ }^{3}$ \\ William Hernán Bolívar-García ${ }^{4}$
}

Fecha de recepción: 7 de junio de 2019

Fecha de aprobación: 15 de agosto de 2019

\section{Resumen}

En este trabajo se buscó identificar las condiciones y variables óptimas de operación para el procesamiento de un mineral de oro, de tal forma que se alcanzara una alta eficiencia en la recuperación del metal precioso. Inicialmente se llevó a cabo una caracterización del mineral en estudio con la realización de diferentes ensayos de laboratorio. Igualmente se ejecutó un ensayo de moliendabilidad. A la vez, una muestra de mineral fue clasificada mediante una serie de tamices Tyler. Después, el material fue sometido a concentración gravimétrica en mesa Wilfley. Los finos, producto de la concentración, con una granulometría 100 \% pasante 170 mallas Tyler, fueron sometidos a pruebas de flotación convencional. De esta forma, con el fin de optimizar la recuperación del oro del mineral aurífero en estudio, se aplicó un diseño experimental de análisis factorial $2 \mathrm{k}$ con punto central, tres variables y el método de superficie de respuesta para determinar los parámetros óptimos con el fin de lograr una recuperación eficiente durante la cianuración. Los resultados obtenidos mostraron que el mineral presenta un alto grado de lixiviabilidad en cianuro, lográndose una recuperación del oro del 89.35 \%. De acuerdo con esto, se corroboró que el diseño experimental estadístico es una herramienta valiosa para el procesamiento eficiente de los minerales, dado que permite encontrar los parámetros óptimos del proceso de cianuración, como son el tiempo de lixiviación, la granulometría

\footnotetext{
${ }^{1}$ M.Sc. Universidad de Antioquia (Medellín-Antioquia, Colombia). jairo.ruiz@udea.edu.co ORCID: 0000-00031086-1317.

${ }_{2}$ M.Sc. Universidad de Antioquia (Medellín-Antioquia, Colombia). ORCID: 0000-0002-1068-2536.

${ }^{3}$ Universidad de Antioquia (Medellín-Antioquia, Colombia). ORCID: 0000-0003-1969-3647.

${ }^{4}$ Universidad de Antioquia (Medellín-Antioquia, Colombia). ORCID: 0000-0001-6261-1323.
} 
del mineral y la concentración de NaCN. Los resultados conseguidos con la aplicación del diseño estadístico en las pruebas de cianuración posibilitaron determinar las condiciones óptimas de operación para una máxima recuperación de oro.

Palabras clave: análisis estadístico; cianuros; flotación; metales preciosos; minerales; oro.

\section{Statistical Modeling and Optimization of the Cyanidation Process of Gold Mineral}

\section{Abstract}

In this paper, we have sought to identify the conditions and optimal operating variables for the processing of a gold ore, in such a way to achieve high efficiency in the recovery of the precious metal. Initially a characterization of the mineral under study was carried out with the performance of different laboratory tests. Likewise, a grinding test was made. In the same way, a sample of ore was classified using a series of Tyler sieves. Next, the material was subjected to gravimetric concentration on a Wilfley concentrator table. The fines, product of the concentration, with a $100 \%$ granulometry through 170 Tyler meshes, were subjected to conventional flotation tests. In this way, to optimize the recovery of gold from the gold ore under study, an experimental design of $2 \mathrm{k}$ factorial analysis with central point, three variables and the response surface method was applied to determine the optimal parameters to achieve an efficient recovery during cyanidation. The results obtained show that the mineral has a high degree of cyanide leachability, obtaining a gold recovery of $89.35 \%$. The research demonstrated that the statistical experimental design is a valuable tool for the efficient processing of the minerals, since it allows finding the optimal parameters of the cyanidation process such as the leaching time, the granulometry of the mineral and the concentration of $\mathrm{NaCN}$. The results obtained with the application of the statistical design in the cyanidation tests allowed determining the optimal operating conditions for a maximum gold recovery.

Keywords: cyanides; floatation; gold; minerals; precious metals; statistical analysis.

\section{Modelamento estatístico e otimização do processo de cianetação de um mineral aurífero}

\section{Resumo}


Neste trabalho buscou-se identificar as condições e variáveis ótimas de operação para o processamento de um mineral de ouro, de tal forma que se alcançará uma alta eficiência na recuperação do metal precioso. Inicialmente foi realizada uma caracterização do mineral em estudo com a realização de diferentes ensaios de laboratório. Igualmente executou-se um ensaio de moagem. Ao mesmo tempo, uma amostra de mineral foi classificada mediante uma série de crivos Tyler. Depois, o material foi submetido a concentração gravimétrica em mesa Wilfley. Os finos, produto da concentração, com uma granulometria $100 \%$ passante 170 malhas Tyler, foram submetidos a provas de flotação convencional. Desta forma, com o fim de otimizar a recuperação do ouro do mineral aurífero em estudo, aplicou-se um desenho experimental de análise fatorial $2 \mathrm{k}$ com ponto central, três variáveis e o método de superfície de resposta para determinar os parâmetros ótimos com o fim de lograr uma recuperação eficiente durante a cianetação. Os resultados obtidos mostraram que o mineral apresenta um alto grau de lixiviabilidade em cianeto, logrando-se uma recuperação do ouro de 89.35 \%. De acordo com isto, corroborou-se que o desenho experimental estatístico é uma ferramenta valiosa para o processamento eficiente dos minerais, dado que permite encontrar os parâmetros ótimos do processo de cianetação, como são o tempo de lixiviação, a granulometria do mineral e a concentração de $\mathrm{NaCN}$. Os resultados conseguidos com a aplicação do desenho estatístico nas provas de cianetação possibilitaram determinar as condições ótimas de operação para uma máxima recuperação do ouro.

Palavras chave: análise estatístico; cianetos; flutuação; metais preciosos; minerais; ouro.

\section{Para citar este artículo:}

J. A. Ruiz-Córdoba, C. A. López-Cañas, M. E. Carmona-Arango, and W. H. BolívarGarcía, "Modelamiento estadístico y optimización del proceso de cianuración de un mineral aurífero," Revista Facultad de Ingeniería, vol. 28 (53), pp. 33-51, Oct. 2019. https://doi.org/10.19053/01211129.v28.n53.2019.9745.

\section{Esta obra está bajo licencia internacional Creative Commons Reconocimiento 4.0}

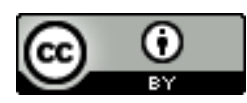




\section{INTRODUCCIÓN}

Uno de los propósitos de la metalurgia extractiva del oro consiste en separar los metales de particular interés, de tal forma que se puedan emplear para un fin determinado, para lo cual existen diversos procedimientos, como la lixiviación con cianuro. Con respecto a este proceso, Santamaría et al. condujeron un estudio acerca de su desempeño al emplear la técnica de cianuración con un mineral aurífero usando como agentes lixiviantes la tiourea y el cianuro sódico [1]. Los resultados mostraron que la lixiviación con tiourea arrojó un mejor rendimiento que la conducida con cianuro, aunque no se aplicó un diseño estadístico experimental para optimizar las variables de operación. Dependiendo de la composición química y mineralógica se encuentran diversos tratamientos para la recuperación del oro, como concentración gravimétrica, flotación, aireación con cal, tostación, calcinación, entre otros [2]. Además, una correcta caracterización mineralógica, fisicoquímica y metalúrgica de los minerales permite plantear los procesos y parámetros de beneficio más convenientes para obtener una mayor recuperación del oro [3].

De esta forma se han estudiado diferentes métodos para el beneficio de minerales auríferos, como son la lixiviación con cloro, tiourea, o tiosulfato, así como la biooxidación $[4,5]$. Otra alternativa a esta última técnica cuando se procesa un mineral refractario con sulfuros, consiste en realizar una oxidación doble, con etapa de lixiviación férrica a alta temperatura y una etapa subsiguiente de biooxidación [6]. Por otro lado, en algunos minerales refractarios de oro y plata, la técnica de la cianuración no es efectiva, por lo que se han llevado a cabo ensayos con tratamientos previos con hidróxido de potasio para mejorar ciertas propiedades del mineral en cuestión a fin de aumentar la extracción del oro [7]. Igualmente, es conocido que en minerales auríferos con altos contenidos de cobre se reduce la cantidad de oro que se podría obtener con la cianuración [8]. La lixiviación de minerales auríferos se encuentra influenciada por diversos factores de orden físico y químico que pueden interferir en la eficiencia de la recuperación de los metales preciosos como el oro y la plata [9]. En el proceso se 
Jairo Antonio Ruiz-Córdoba, Carlos Alberto López-Cañas, María Eugenia Carmona-Arango, William Hernán

utiliza el oxígeno del aire como oxidante y al ion $\mathrm{CN}$ - como agente complejante. Esta técnica se basa en la circunstancia de que el oro se disuelve fácilmente en una solución diluida de cianuro de sodio o de potasio y con relativa facilidad si se mantienen condiciones oxidantes favorables [10]. Para esto es apropiado utilizar el diseño de experimentos como herramienta para la planificación y el análisis de las pruebas metalúrgicas con las cuales se realizan cambios programados en los parámetros de control de un proceso, a fin de observar e identificar los efectos de estos en la variable respuesta [11].

El diseño experimental planteado es el de análisis factorial $2 \mathrm{k}$, con punto central aplicado con tres variables, empleando la metodología de superficie de respuesta (MSR) con la finalidad de determinar el rendimiento de las pruebas de cianuración en botella por agitación sobre rodillos. En esta investigación se establecieron como variables de control la granulometría del mineral, la concentración de cianuro de sodio y el tiempo de lixiviación; como variable respuesta se definió la recuperación del oro. El diseño experimental fue planteado y analizado con ayuda del software Statgraphics [12].

Figueredo et al. [13] realizaron una caracterización química, mineralógica y granulométrica de una muestra de mineral de oro, para luego llevar a cabo las pruebas de lixiviación, sin aplicar un diseño experimental estadístico. Otros investigadores han utilizado de manera novedosa ciertos elementos ya conocidos, como el carbón activado, para mejorar la eficiencia de la cianuración del mineral de oro, y han mostrado resultados interesantes; sin embargo, no aplican el diseño estadístico experimental [14]. Azañero et al. [15] muestran las dificultades para el beneficio de minerales con pirita y las bajas tasas de recuperación de oro que se pueden presentar con los métodos convencionales de lixiviación con cianuro. No plantean la ejecución de un diseño experimental estadístico.

Igualmente, se han llevado a cabo ensayos para mejorar la cinética de la cianuración a través de cambios en parámetros de la moliendabilidad sin la aplicación del diseño experimental estadístico [16]. Khosravi et al. estudiaron la adsorción del oro en soluciones lixiviadas de cianuro, para lo cual definieron un 
análisis estadístico factorial lineal, de interacción factorial cuadrática y cúbica, para describir el comportamiento de diversos factores del proceso. Igualmente, aplicaron ANOVA y obtuvieron superficies de respuesta en 3D. El uso de este diseño experimental fue útil para mejorar las tasas de adsorción de oro [17]. De la misma forma, Korolev et al. [18] aplicaron un diseño estadístico factorial para incrementar la recuperación de oro presente en pequeñas cantidades de soluciones cianuradas libres de cloro. Se evaluaron el coeficiente de determinación $\mathrm{R}^{2}$, el coeficiente de modelo de error estándar y el análisis de variancia ANOVA. Los resultados alcanzados fueron satisfactorios.

Si bien se muestra un trabajo investigativo que mejora los procesos de cianuración y que en algunos proyectos se llevaron a cabo desarrollos estadísticos interesantes, se puede apreciar que este trabajo presenta una metodología más estructurada con respecto a los reportados en la literatura. Así mismo, los resultados alcanzados permitieron conocer un amplio conjunto de parámetros que se pueden optimizar con la aplicación de un diseño experimental de análisis factorial, como fueron la granulometría del material, la concentración y consumo de $\mathrm{NaCN}$, el consumo de $\mathrm{CaO}$ y el tiempo de cianuración.

\section{Metodología}

En la Figura 1 se presenta el diagrama de flujo de las operaciones y procesos utilizados en la investigación.

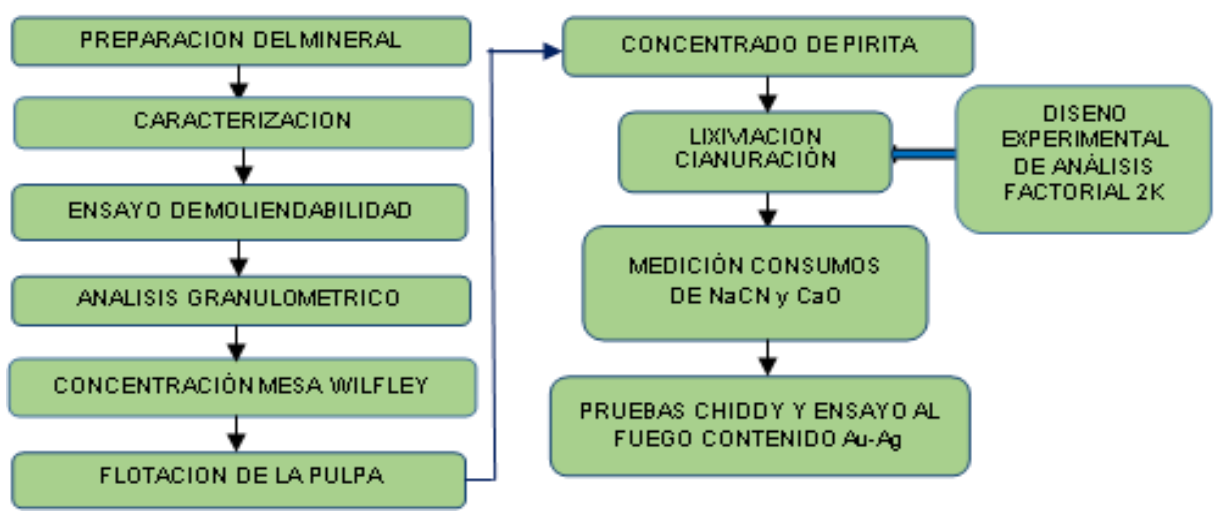

Fig. 1. Diagrama de flujo de las operaciones y procesos. 


\section{A. Muestreo y preparación del material}

El mineral aurífero de estudio contiene sulfuros de veta proveniente de la vereda Santa Cruz del municipio de Remedios, localizado en el nordeste del departamento de Antioquia, del cual se obtuvo una muestra representativa de 120 $\mathrm{kg}$ de mineral.

\section{B. Caracterización del mineral}

La muestra mineral fue analizada por fluorescencia de rayos $X(F R X)$, microscopia estereoscópica y difracción de rayos X (DRX).

Se ejecutó un ensayo de moliendabilidad con el objeto de analizar la variación en la reducción del tamaño del mineral con el tiempo y lograr estimar el tiempo requerido de molienda para obtener una determinada granulometría. El material empleado fue triturado hasta obtener un D80 de $1653 \mu \mathrm{m}$ y un D50 de $1283 \mu \mathrm{m}$. Se efectuó análisis granulométrico del mineral a 5, 10, 15, 20, 30 y 40 minutos de molienda. En la Tabla 1se presentan las condiciones utilizadas en el ensayo de moliendabilidad.

Tabla 1. Parámetros usados en el ensayo de moliendabilidad del mineral.

\begin{tabular}{|c|c|c|c|}
\hline Equipo & Parámetros & \multicolumn{2}{|c|}{ Valor } \\
\hline \multirow{3}{*}{$\begin{array}{l}\text { Especificaciones del } \\
\text { molino }\end{array}$} & Longitud del molino & \multicolumn{2}{|c|}{$20 \mathrm{~cm}$} \\
\hline & Diámetro interno del molino & \multicolumn{2}{|c|}{$19 \mathrm{~cm}$} \\
\hline & Volumen interno del molino & \multicolumn{2}{|c|}{$5670.6 \mathrm{~cm}^{3}$} \\
\hline \multirow{7}{*}{$\begin{array}{l}\text { Parámetros } \\
\text { operativos }\end{array}$} & Velocidad de rotación del molino & \multicolumn{2}{|c|}{$76.3 \mathrm{rpm}$} \\
\hline & Volumen de llenado del molino & \multicolumn{2}{|c|}{$35 \%$} \\
\hline & Carga mineral & \multicolumn{2}{|c|}{$3288.3 \mathrm{~g}$} \\
\hline & \multirow{4}{*}{$\begin{array}{l}\text { Distribución de los cuerpos } \\
\text { moledores (bolas) }\end{array}$} & Diámetro $(\mathrm{cm})$ & Cantidad \\
\hline & & 1.7 & 82 \\
\hline & & 2.5 & 39 \\
\hline & & 3.9 & 15 \\
\hline
\end{tabular}

Para obtener el concentrado de pirita para la lixiviación, la muestra de mineral fue clasificada con tamices Tyler $-35+80,-80+140$ y $-140+170$ mallas, y sometida a concentración gravimétrica en mesa Wilfley. Los finos resultantes con una granulometría $100 \%$ pasante 170 mallas Tyler fueron sometidos a pruebas de 
flotación convencional, empleando una pulpa con el 40 \% de sólidos en celda de flotación tipo laboratorio. En la Tabla 2 se presentan los parámetros de operación de la mesa. La Tabla 3 muestra los reactivos y dosificación usada en el ensayo de flotación, con base en información hallada en la literatura [19, 20].

Tabla 2. Parámetros operativos usados en la concentración en mesa Wilfley.

\begin{tabular}{|c|c|c|}
\hline Mallas & Angulo de inclinación $(\boldsymbol{\theta})$ & Flujo de Agua (gal/min) \\
\hline$-35+80$ & 17.5 & 2.5 \\
\hline$-80+140$ & 16 & 2.3 \\
\hline$-140+170$ & 16 & 2 \\
\hline
\end{tabular}

Tabla 3. Reactivos y dosificación usados en la flotación del mineral.

\begin{tabular}{|c|c|c|}
\hline Reactivo & Función & Dosificación (g/ton) \\
\hline Aerofloat 31 (A31) & Promotor & 50 \\
\hline Aerofroth 65 (A65) & Espumante & 20 \\
\hline Xantato amilico de potasio & Colector & 25 \\
\hline
\end{tabular}

\section{Diseño experimental factorial $2 k$ con punto central en pruebas de cianuración}

En las pruebas de lixiviación de cianuración en botella por agitación sobre rodillos, se utilizó el diseño experimental de análisis factorial $2 \mathrm{k}$ con un punto central adicional que permite detectar la no linealidad de la variable respuesta, e identificado en tres variables con el objeto de optimizar el proceso. Para ello se empleó la metodología de superficie de respuesta (MSR), a fin de determinar el efecto de las variables e interacciones entre estas sobre la variable respuesta.

La Tabla 4 ilustra las condiciones de operación usadas en las pruebas de cianuración, mientras que la Tabla 5 presenta las variables y niveles considerados en el diseño experimental en las pruebas de cianuración en botella rotatoria.

Tabla 4. Condiciones usadas en pruebas de cianuración en botella.

\begin{tabular}{|c|c|}
\hline Condición & Valor \\
\hline Densidad de pulpa (\% solidos) & 33 \\
\hline Masa mineral concentrado (g) & 500 \\
\hline Volumen pulpa (ml) & 1515 \\
\hline Acidez (pH), controlado con Cao & $10.5-11.5$ \\
\hline Velocidad rotación molino (rpm) & 100 \\
\hline
\end{tabular}


Jairo Antonio Ruiz-Córdoba, Carlos Alberto López-Cañas, María Eugenia Carmona-Arango, William Hernán

Tabla 5. Variables y niveles estudiados en el diseño experimental para las pruebas de cianuración.

\begin{tabular}{|c|c|c|c|}
\hline Factor & Nivel Bajo & Nivel Medio & Nivel Alto \\
\hline Concentrado NaCN $\left(\mathrm{Lb} / \mathrm{m}^{3}\right)$ & 1 & 1.75 & 2.5 \\
\hline Granulometría (mallas) & -140 & -200 & -270 \\
\hline Tiempo (horas) & 3 & 7.5 & 12 \\
\hline
\end{tabular}

\section{Resultados}

\section{A. Caracterización del mineral}

La Tabla 6 muestra la composición aproximada de las especies mineralógicas presentes en el mineral de cabeza.

Tabla 6. Caracterización microscópica del mineral.

\begin{tabular}{|c|c|}
\hline Especies mineralógicas & Composición (\%) \\
\hline Cuarzo & 90 \\
\hline Pirita y otros sulfuros & 5 \\
\hline Arcillas y otros silicatos & 3 \\
\hline Magnetita (Fe3O4) & 1 \\
\hline Hematita & Trazas (<1) \\
\hline
\end{tabular}

La Figura 2 representa el difractograma obtenido por DRX, corroborándose que el mineral está constituido principalmente por cuarzo $\left(\mathrm{SiO}_{2}\right)$ y pirita $\left(\mathrm{FeS}_{2}\right)$.

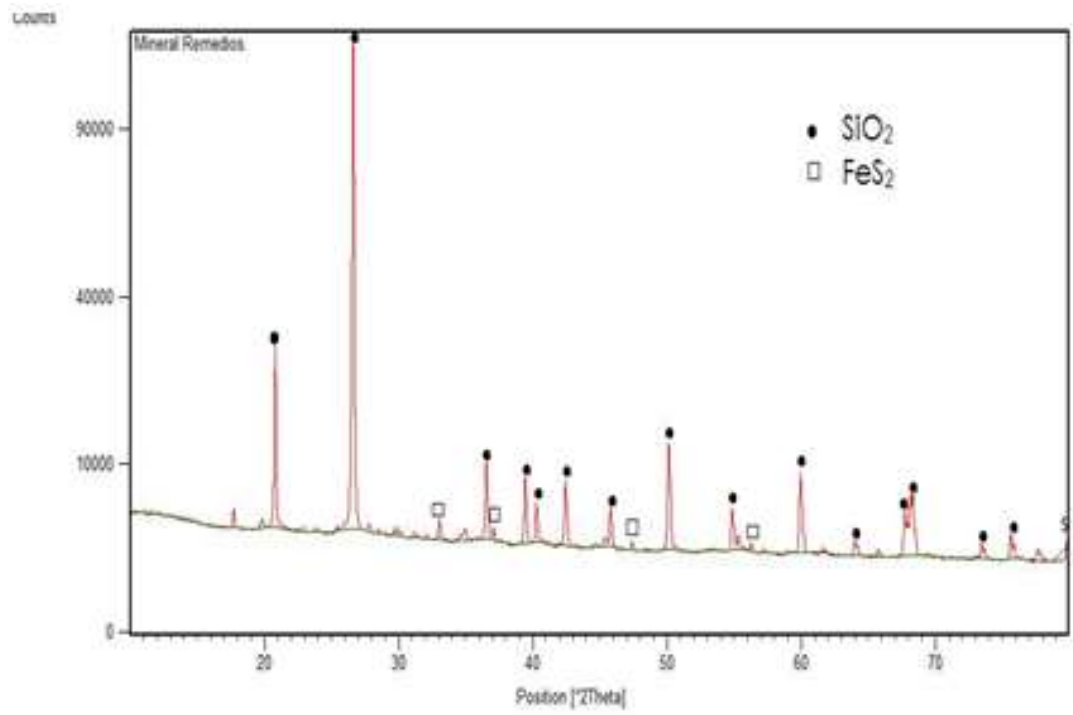

Fig. 2. Difractograma de Rayos $X$ del mineral de cabeza. 
La Tabla 7 presenta los resultados de tenores de Au y Ag del mineral de cabeza, obtenidos por ensayo al fuego.

Tabla 7. Tenores de Au y Ag en el mineral de cabeza.

\begin{tabular}{|c|c|c|c|c|c|c|}
\hline Muestras & $\begin{array}{c}\text { Peso botón de } \\
\text { plomo (g) }\end{array}$ & Peso Doré & Peso Au (g) & Peso Ag (g) & $\begin{array}{c}\text { Tenor Au } \\
(\mathbf{g} / \mathbf{t})\end{array}$ & $\begin{array}{c}\text { Tenor Ag } \\
(\mathbf{g} / \mathbf{t})\end{array}$ \\
\hline 1 & 27.42 & 0.00171 & 0.00171 & 0.00011 & 5.5 & 80 \\
\hline 2 & 27.92 & 0.00180 & 0.00010 & 0.0017 & 5 & 85 \\
\hline Promedio & 27.67 & 0.0011755 & 0.000105 & 0.00165 & 5.25 & 82.5 \\
\hline
\end{tabular}

La Figura 3 representa los resultados de la distribución por fracciones granulométricas de Au y Ag en el mineral de cabeza.

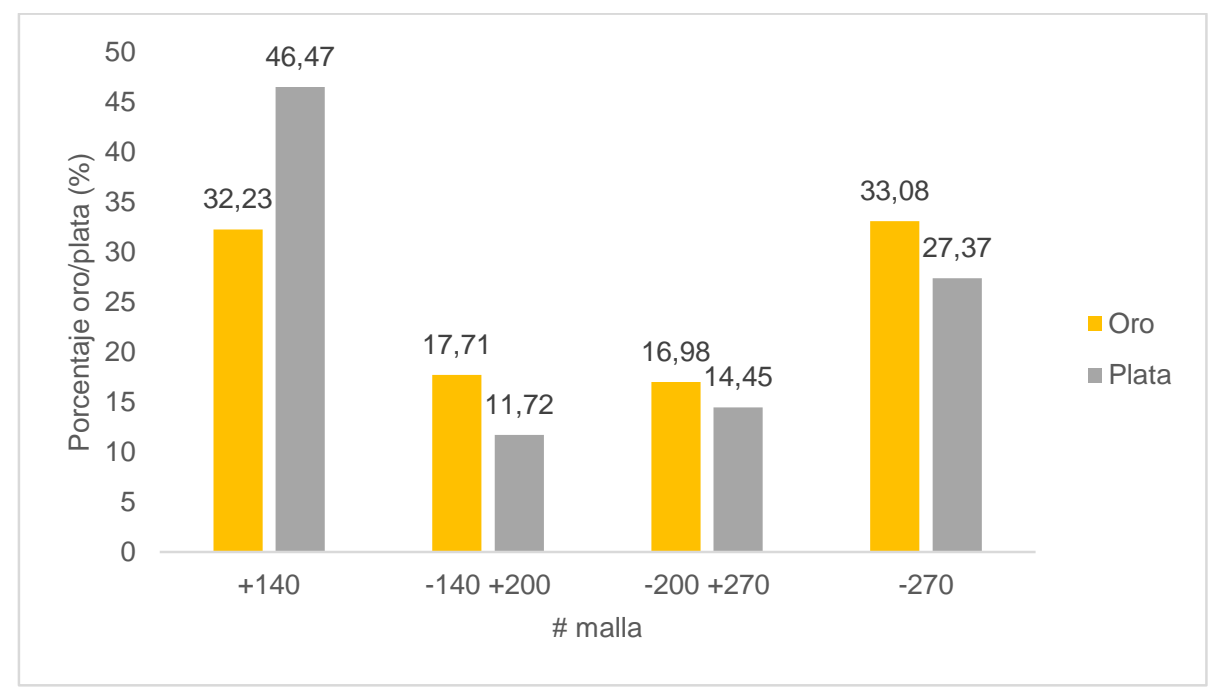

Fig. 3. Distribución de Au y Ag del mineral por fracciones granulométricas.

Puede observarse que el $50.06 \%$ de $\mathrm{Au}$ y $41.82 \%$ de $\mathrm{Ag}$ se presentan en la fracción fina -200 mallas $(-75 \mu \mathrm{m})$, tamaños apropiados para cianuración; presentando el mayor contenido de $\mathrm{Au}$ y $\mathrm{Ag}$ del $33.08 \%$ y $27.37 \%$, respectivamente, en la fracción más fina -270 mallas $(-53 \mu \mathrm{m})$. El $49.94 \% \mathrm{Au}$ y el $58.18 \%$ Ag se presentan entre las fracciones gruesas +140 mallas $(+105 \mu \mathrm{m}) \mathrm{y}$ 140+200 mallas (-105 a $75 \mu \mathrm{m}$ ), tamaños no muy adecuados para la cianuración.

La Figura 4 muestra la curva de moliendabilidad o de variación del D80 del mineral, obtenida en los tiempos de molienda de $5,10,15,20,30$ y 40 minutos. 
Para esta línea de tendencia se tuvo un ajuste en la descripción del comportamiento del $96.58 \%$. La Tabla 8 muestra los índices metalúrgicos de recuperación del oro del mineral por los métodos de concentración en mesa y flotación.

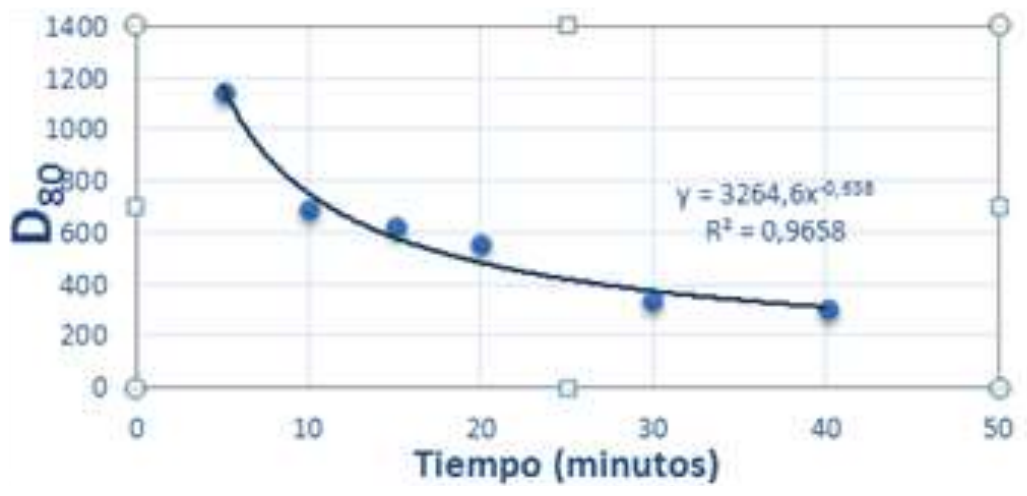

Fig. 4. Curva de moliendabilidad del mineral.

Tabla 8. Índices metalúrgicos obtenidos en pruebas de concentración en mesa y flotación.

\begin{tabular}{|c|c|c|c|c|}
\hline Índice Metalúrgico & $\begin{array}{c}\text { Mesa } \\
-35+80 \\
\text { mallas }\end{array}$ & $\begin{array}{c}\text { Mesa } \\
-80+140 \\
\text { mallas }\end{array}$ & $\begin{array}{c}\text { Mesa } \\
-140+170 \\
\text { mallas }\end{array}$ & $\begin{array}{c}\text { Flotación } \\
-170 \\
\text { mallas }\end{array}$ \\
\hline Recuperación de oro (\%) & 80 & 89.2 & 79.2 & 85 \\
\hline Razón de enriquecimiento & 3.2 & 7.4 & 1.9 & 3.6 \\
\hline
\end{tabular}

\section{B. Diseño Experimental $2 \boldsymbol{k}$ con punto central en la lixiviación}

1) Experimentos realizados. Según el diseño experimental planteado, factorial $2 \mathrm{k}$, de tres variables, dos niveles y punto central, serían necesarios 27 experimentos, pero mediante el software Statgraphics 16.0, estos se lograron reducir a 16 con una confiabilidad en los resultados del $93 \%$. La Tabla 9 muestra el diseño experimental planteado.

Tabla 9. Diseño experimental y resultados obtenidos en las pruebas de cianuración en botella.

\begin{tabular}{|c|c|c|c|c|}
\hline Ensayos & Tiempo(horas) & $\begin{array}{c}\text { Granulometría } \\
\text { (mallas) }\end{array}$ & $\begin{array}{c}\text { Concentración NaCN } \\
\left(\mathbf{L b} / \mathbf{m}^{\mathbf{3}} \text { ) }\right.\end{array}$ & $\begin{array}{c}\text { Recuperación Oro } \\
(\mathbf{\%})\end{array}$ \\
\hline 1 & 3 & 270 & 2.50 & 83.20 \\
\hline 2 & 3 & 270 & 1.00 & 68.48 \\
\hline 3 & 3 & 140 & 2.50 & 76.48 \\
\hline
\end{tabular}




\begin{tabular}{|c|c|c|c|c|}
\hline Ensayos & Tiempo(horas) & $\begin{array}{l}\text { Granulometría } \\
\text { (mallas) }\end{array}$ & $\begin{array}{c}\text { Concentración NaCN } \\
\left(\mathrm{Lb} / \mathrm{m}^{3}\right)\end{array}$ & $\begin{array}{c}\text { Recuperación Oro } \\
(\%)\end{array}$ \\
\hline 4 & 7.5 & 270 & 1.75 & 78.40 \\
\hline 5 & 12 & 270 & 2.50 & 72.00 \\
\hline 6 & 12 & 200 & 1.75 & 86.40 \\
\hline 7 & 3 & 200 & 1.75 & 73.28 \\
\hline 8 & 12 & 140 & 2.50 & 81.76 \\
\hline 9 & 7.5 & 200 & 2.50 & 89.60 \\
\hline 10 & 3 & 140 & 1.00 & 60.96 \\
\hline 11 & 12 & 140 & 1.00 & 71.52 \\
\hline 12 & 7.5 & 200 & 1.75 & 83.68 \\
\hline 13 & 7.5 & 200 & 1.00 & 81.76 \\
\hline 14 & 12 & 270 & 1.00 & 82.40 \\
\hline 15 & 7.5 & 200 & 1.75 & 80.48 \\
\hline 16 & 7.5 & 140 & 1.75 & 72.80 \\
\hline
\end{tabular}

2) ANOVA para la recuperación de oro. La Tabla 10 presenta el resultado del ANOVA que permite conocer cuáles de las variables e interacciones entre estas tienen efectos significativos sobre la variable respuesta o recuperación del oro en las pruebas de lixiviación con cianuro.

Tabla 10. ANOVA para la recuperación de oro.

\begin{tabular}{|l|c|c|c|c|c|}
\hline \multicolumn{1}{|c|}{ Fuente } & Suma de Cuadrados & GI & Cuadrado Medio & Razón-F & Valor-P \\
\hline A:tiempo & 98,6797 & 1 & 98,6797 & 7,04 & 0,0379 \\
\hline B:granulometría & 43,9322 & 1 & 43,9322 & 3,13 & 0,1271 \\
\hline C:concentración & 140,642 & 1 & 140,642 & 10,03 & 0,0194 \\
\hline AA & 27,2764 & 1 & 27,2764 & 1,94 & 0,2126 \\
\hline AB & 23,235 & 1 & 23,235 & 1,66 & 0,2455 \\
\hline AC & 115,52 & 1 & 115,52 & 8,24 & 0,0284 \\
\hline BB & 152,752 & 1 & 152,752 & 10,89 & 0,0164 \\
\hline BC & 57,4968 & 1 & 57,4968 & 4,10 & 0,0893 \\
\hline CC & 18,1447 & 1 & 18,1447 & 1,29 & 0,2987 \\
\hline Error total & 84,148 & 6 & 14,0247 & & \\
\hline Total (corr.) & 827,539 & 15 & & & \\
\hline
\end{tabular}

R-cuadrada (ajustada por G.I.) $=74.5788 \%$; Error estándar del estudio $=3.74495$; Error absoluto medio $=$ 1.97959; Estadístico Durbin-Watson $=3.10262(P=0,9901)$; Autocorrelación residual de Lag $1=-0.605272$

El valor R-cuadrada es $89.8315 \%$ e indica que el modelo se ajusta bien a los datos. Los valores $-\mathrm{P}$ menores a 0.05 indican que las variables tienen un efecto significativo en la recuperación del oro con un nivel de confianza del $95 \%$. 


\section{Balance metalúrgico}

1) Concentración en mesa Wilfley. Los resultados de la concentración en mesa Wilfley para la fracción de tamaño - $80+140$ se presentan en las Tablas 11 y 12 .

Tabla 11. Masas y tenores de los materiales en la concentración en mesa.

\begin{tabular}{|l|c|c|c|}
\hline \multicolumn{1}{|c|}{ Material } & Masas (gr) & Masas (T) & Tenor oro (gr/TM) \\
\hline Alimento (A) & 1000 & 0.001 & 5.25 \\
\hline Concentrado (C) & 120 & 0.00012 & 38.85 \\
\hline Colas (T) & 880 & 0.00088 & 0,67 \\
\hline
\end{tabular}

Tabla 12. Índices metalúrgicos de la concentración en mesa Wilfley.

\begin{tabular}{|l|c|}
\hline \multicolumn{1}{|c|}{ Parámetros } & Valores obtenidos \\
\hline Recuperación $(\mathrm{R}) \%$ & 89.20 \\
\hline Razón enriquecimiento (RE) & 7.4 \\
\hline Rendimiento $(\mathrm{V}) \%$ & 12 \\
\hline Razón de concentración $(\mathrm{RC})$ & 8.33 \\
\hline
\end{tabular}

\section{2) Balance másico.}

$$
\begin{gathered}
\mathbf{A}=\mathbf{C}+\mathbf{T} \longrightarrow \begin{array}{l}
1000 \mathrm{gr}=120 \mathrm{~g}+880 \mathrm{gr} \\
1000 \mathrm{gr}=1000 \mathrm{gr}
\end{array}
\end{gathered}
$$

\section{3) Balance metalúrgico.}

$\mathrm{F}\left(\mathrm{t}_{\mathrm{f}}\right)=\mathrm{C}\left(\mathrm{t}_{\mathrm{c}}\right)+\mathrm{T}(\mathrm{t} \mathrm{T})$

$1000 \mathrm{gr} \times 5.25 \mathrm{gr} / \mathrm{TM}=120 \mathrm{~g} \times 38.85 \mathrm{gr} / \mathrm{TM}+880 \mathrm{TM} \times 0.668 \mathrm{gr} / \mathrm{TM}$

$5250 \mathrm{gr}=4662 \mathrm{gr}+587.84 \mathrm{gr}$

$5250 \mathrm{gr}=5249.84 \mathrm{gr}$

4) Concentración por flotación. Los resultados de la concentración por flotación para la fracción de tamaño -170 mallas Tyler se muestran en las Tablas 13 y 14 .

Tabla 13. Masas y tenores de los materiales en la concentración por flotación.

\begin{tabular}{|l|c|c|c|}
\hline \multicolumn{1}{|c|}{ Material } & Masas(gr) & Masas(T) & Tenor oro (gr/Ton) \\
\hline Alimento (A) & 1000 & 0.001 & 5.25 \\
\hline Concentrado (C) & 230 & 0.000230 & 18.9 \\
\hline Colas & 770 & 0.000877 & 1.17 \\
\hline
\end{tabular}


Tabla 14. Índices metalúrgicos de la concentración por flotación.

\begin{tabular}{|l|c|}
\hline \multicolumn{1}{|c|}{ Parámetros } & Valores obtenidos \\
\hline Recuperación $(\mathrm{R}) \%$ & 85 \\
\hline Razón enriquecimiento (RE) & 3.6 \\
\hline Rendimiento (V) \% & 23.6 \\
\hline Razón de concentración $(\mathrm{RC})$ & 4.23 \\
\hline
\end{tabular}

\section{5) Balance.}

$\mathrm{F}(\mathrm{tf})=\mathrm{C}\left(\mathrm{t}_{\mathrm{c}}\right)+\mathrm{T}(\mathrm{t} \mathrm{T})$

$1000 \mathrm{~g} \times 5.25 \mathrm{gr} . / \mathrm{TM}=230 \mathrm{~g} \times 18.9 \mathrm{gr} . / \mathrm{TM}+770 \mathrm{~g} \times 1.172 \mathrm{gr} . / \mathrm{TM}$

5250 gr. $=4347$ gr. +902.44 gr.

5250 gr. $=5249.44$ gr.

\section{Discusıón}

\section{A. Figuras de superficie de respuesta y contorno de la superficie de respuesta estimadas}

Las figuras 5 y 6 representan la superficie de respuesta y el contorno de la superficie de respuesta, estimadas para una granulometría de 200 mallas Tyler $(75 \mu \mathrm{m})$. En estas se corrobora la relevancia de las variables establecidas como principales a partir de ANOVA.

En la Figura 5, de superficie de respuesta, se observa que es posible obtener recuperaciones entre $89-93 \%$ de $A u$ con 7 horas de cianuración y una concentración de $2.5 \mathrm{lb}$ de $\mathrm{NaCN} / \mathrm{m}^{3}$ de solución. De acuerdo con la Figura 6 de contorno de la superficie de respuesta, se obtiene la máxima recuperación del oro, $89.35 \%$, a $2.5 \mathrm{lb}$ de $\mathrm{NaCN} / \mathrm{m}^{3}$ y siete horas de lixiviación. 


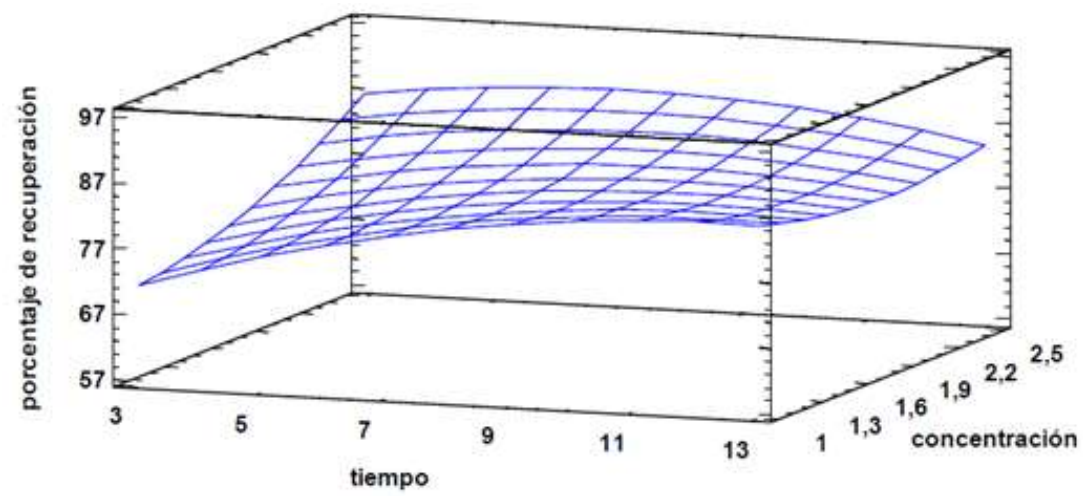

Fig. 5. Superficie de respuesta estimada para una granulometría de 200 mallas Tyler.

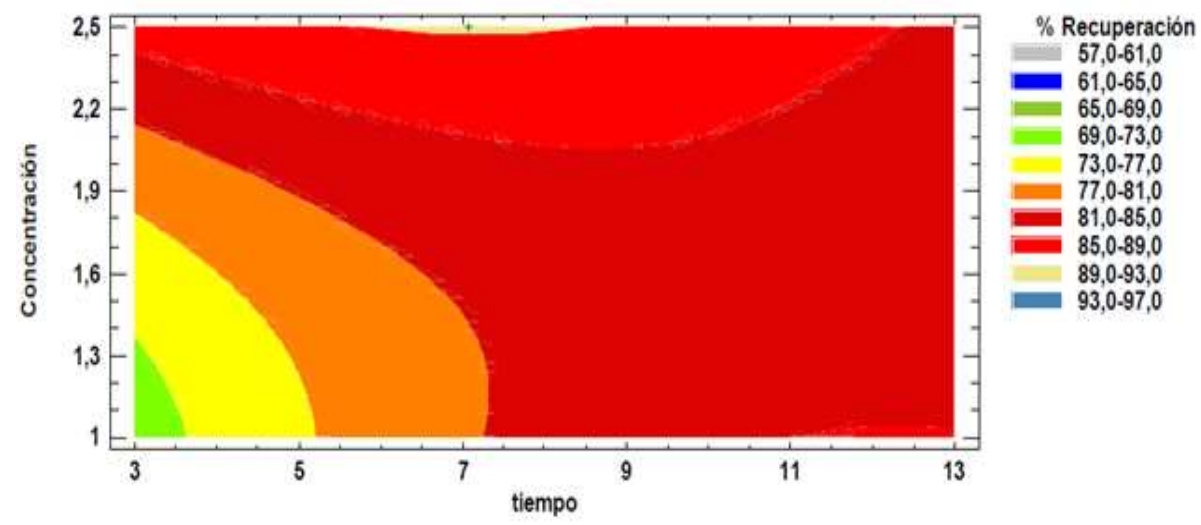

Fig. 6. Contorno de la superficie de respuesta estimada para una granulometría de 200 mallas

Tyler.

\section{B. Optimización de la variable respuesta}

En la Tabla 15 se presentan los parámetros óptimos para la lixiviación con cianuro del mineral en estudio, obtenidos con el diseño estadístico de experimentos y el empleo de la metodología de superficie de respuesta en las pruebas de cianuración en botella por agitación sobre rodillos. Con estos parámetros se obtiene una recuperación máxima del oro del $89.35 \%$.

Tabla 15. Parámetros óptimos para la cianuración del mineral, obtenidos con el diseño experimental utilizado.

\begin{tabular}{|c|c|c|}
\hline Parámetros & Óptimo & Real \\
\hline Granulometría mineral (mallas Tyler) & -203.2 & -200 \\
\hline
\end{tabular}




\begin{tabular}{|l|c|c|}
\hline \multicolumn{1}{|c|}{ Parámetros } & Óptimo & Real \\
\hline Concentración de $\mathrm{NaCN}\left(\mathrm{lb} / \mathrm{m}^{3}\right)$ & 2.5 & 2.5 \\
\hline Consumo $\mathrm{NaCN}(\mathrm{Kg} / \mathrm{TM})$ & 1.25 & 1.25 \\
\hline Consumo CaO $(\mathrm{Kg} / \mathrm{TM})$ & 1.5 & 1.5 \\
\hline Tiempo cianuración $(\mathrm{hrs})$ & 7.07 & 7 \\
\hline
\end{tabular}

\section{CONCLUSIONES}

En la concentración por flotación también se obtuvieron buenos resultados. Se logró un porcentaje de recuperación del 85 \% y una razón de enriquecimiento de 3.6, con el material fino, - 170 mallas Tyler $(-88 \mu \mathrm{m})$, producto de la concentración en mesa Wilfley. Según esto, es conveniente implementar procesos de concentración previos a la lixiviación con cianuro a fin de reducir la cantidad de mineral, el consumo energético, de reactivos y de agua en su tratamiento.

Los resultados obtenidos en las pruebas de cianuración en botella por agitación sobre rodillos y el modelo matemático obtenido, permitieron determinar las condiciones óptimas de operación para la máxima recuperación del oro: granulometría del mineral -200 mallas Tyler $(-75 \mu)$, concentración de $\mathrm{NaCN}$ de $2.5 \mathrm{~g} / \mathrm{m}^{3}$ de solución y tiempo de lixiviación de siete horas, con lo cual se obtiene una máxima recuperación del oro del $89.35 \%$.

El diseño experimental aplicado en el beneficio de minerales constituye una adecuada herramienta tecnológica que permite obtener información de calidad a partir del estudio de las variables establecidas y las interacciones potenciales que puedan presentarse entre ellas en un proceso determinado, lo cual es de gran utilidad para lograr comprender mejor un proceso y además facilita la toma oportuna de decisiones respecto a cómo optimizarlo y aumentar su eficiencia.

\section{CONTRIBUCIÓN DE LOS AUTORES}

Los autores contribuyeron en el trabajo de la siguiente forma: el ingeniero Jairo Ruiz Córdoba elaboró el procedimiento de caracterización del mineral y el diseño estadístico, el ingeniero William Bolívar llevó a cabo las diferentes pruebas de laboratorio de caracterización del mineral, y los ingenieros María E. Carmona y 
Jairo Antonio Ruiz-Córdoba, Carlos Alberto López-Cañas, María Eugenia Carmona-Arango, William Hernán

Bolívar-García

Carlos A. López interpretaron las pruebas. El grupo de trabajo completo colaboró en el análisis y discusión de los resultados y las conclusiones.

\section{AGRADECIMIENTOS}

Los autores expresan sus agradecimientos a los directores y técnicos de los laboratorios del Departamento de Ingeniería Metalúrgica y Materiales de la Universidad de Antioquia, por sus encomiables esfuerzos en la realización de las diferentes pruebas al mineral analizado y generar resultados confiables.

\section{REFERENCIAS}

[1] J. Santamaría, R. Torres, M. Parra, and C. Ortiz. "Comparación de cianuro y tiourea como agentes lixiviantes de un mineral aurífero colombiano," Revista Facultad de Ingeniería, vol. 22 (34), pp. 97103, Jun. 2013. https://doi.org/10.19053/01211129.2222.

[2] N. Iglesias, F. Carranza, and I. Palencia, "La biolixiviación como pretratamiento de menas auríferas refractarias en matriz de sulfuros," Revista de Metalurgia, vol. 34 (1), pp. 29-38, Feb. 1998. https://doi.org/10.3989/revmetalm.1998.v34.i1.656.

[3] J. Yánez, I. García, J. Pedraza, and D. Laverde "Caracterización de los minerales auríferos de la zona minera de San Pedro Frío (Bolívar-Colombia), para la selección de los procesos de extracción," Dyna, vol. 72 (145), pp. 22-35, 2005.

[4] B. Xu, Y. Yang, T. Jiang, Q. Li, X. Zhang, and D. Wang, "Improved thiosulfate leaching of a refractory gold concentrate calcine with additives," Hydrometallurgy, vol. 152, pp. 214-222, Feb. 2015. https://doi.org/10.1016/j.hydromet.2014.12.016.

[5] Y. Guo, X. Guo, H. Wu, S. Li, G. Wang, X. Liu, G. Qiu, and D. Wang, "A novel bio-oxidation and twostep thiourea leaching method applied to a refractory gold concentrate," Hydrometallurgy, vol. 171, pp. 213-221, Aug. 2017. https://doi.org/10.1016/i.hydromet.2017.05.023.

[6] M. Muravyov, and A. Bulaev, "Two-step oxidation of a refractory gold-bearing sulfidic concentrate and the effect of organic nutrients on its biooxidation," Minerals Engineering, vol. 45, pp. 108-114, May. 2013. https://doi.org/10.1016/j.mineng.2013.02.007.

[7] I. Alp, O. Celep, D. Paktung, and Y. Thibault, "Influence of potassium hydroxide pretreatment on the extraction of gold and silver from a refractory ore," Hydrometallurgy, vol. 146, pp. 64-71, May. 2014. https://doi.org/10.1016/j.hydromet.2014.03.007.

[8] E. A. Oraby, and J. J. Eksteen, "Gold leaching in cyanide-starved copper solutions in the presence of glycine," Hydrometallurgy, $\quad$ vol. $\quad 156, \quad$ pp. $81-88, \quad$ Jul. 2015. https://doi.org/10.1016/j.hydromet.2015.05.012.

[9] J. Tremolada, Caracterización, influencia y tratamiento de arcillas en procesos de cianuración en pilas, Master Thesis, Universidad de Oviedo, España, 2011, pp. 19-23. 
Modelamiento estadístico y optimización del proceso de cianuración de un mineral aurífero

[10] E. Salinas, I. Rivera, F. Carrillo, F. Patiño, J. Hernández, and L. Hernández, "Mejora del proceso de cianuración de oro y plata, mediante la preoxidación de minerales sulfurosos con ozono," Journal of the Mexican Chemical Society, vol. 48 (4), pp. 315-320.

[11] M. Tanco, and L. Ilzarbe, "Aplicación del Diseño de Experimentos (DOE) para la mejora de procesos," Memorias, vol. 6, pp. 85-94, 2008.

[12] J. Chavarriaga, and J. Giraldo. Estudio estadístico de la lixiviación con cianuro como proceso alternativo a la amalgamación y valoración del ambiente laboral de una muestra de entables y compras de oro del municipio de Remedios Antioquia, Grade Thesis, Universidad de Antioquia, Colombia, 2014, pp. 33-49.

[13] M. Figueredo, "Lixiviación por cianuración de minerales preciosos de un yacimiento en la zona oriental de Cuba," Infomin, vol. 6 (2), pp. 3-12, 2014.

[14] P. Navarro, and C. Vargas, "Efecto de las propiedades físicas del carbón activado en la adsorción de oro desde medio cianuro," Revista de Metalurgia, vol. 46 (3), 227-239, Jun. 2010. https://doi.org/10.3989/revmetalm.0929.

[15] A. Azañero, V. Aramburú, J. Quiñones, L. Puente, M. Cabrera, V. Falconí, J. Quispe, O. F. Cardoza, K. Jaimes, and A. Medina., "Tratamiento hidrometalúrgico del oro diseminado en pirita y arsenopirita del relave de flotación," Revista del Instituto de Investigaciones FIGMMG, vol. 13 (25), pp. 7-12, 2010.

[16] E. Barzola, B. Barzola, D. Lovera, and V. Arias, "Efecto de la moliendabilidad en la cinética de la cianuración de un preconcentrado de Au-Cu," Revista del Instituto de Investigaciones FIGMMG, vol. 14 (27), pp. 47-52, 2011.

[17] R. Khosravi, A. Azizi, R. Ghaedrahmati, V. Kumar, and S. Agarwal, "Adsorption of gold from cyanide leaching solution onto activated carbon originating from coconut shell-Optimization, kinetics and equilibrium studies," Journal of Industrial and Engineering Chemistry, vol. 54, pp. 464-471, Oct. 2017. https://doi.org/10.1016/.jijec.2017.06.036.

[18] I. Korolev, P. Altınkaya, P. Halli, P.-M. Hannula, K. Yliniemi, and M. Lundström, "Electrochemical recovery of minor concentrations of gold from cyanide-free cupric chloride leaching solutions," Journal of Cleaner Production, vol. 186, pp. 840-850, Jun. 2018. https://doi.org/10.1016/j.jclepro.2018.03.177.

[19] A. Teague, J. S. J. Van Deventer, and C. Swaminathan, "A conceptual model for gold flotation," Minerals Engineering, vol. 12 (9), pp. 1001-1019, Sep. 1999. https://doi.org/10.1016/s08926875(99)00087-4.

[20] J. Zhang, S. Shen, Y. Cheng, H. Lan, X. Hu, and F. Wang, "Dual lixiviant leaching process for extraction and recovery of gold from ores at room temperature," Hydrometallurgy, vol. 144-145, pp. 114-123, Apr. 2014. https://doi.org/10.1016/j.hydromet.2014.02.001.

Revista Facultad de Ingeniería (Rev. Fac. Ing.) Vol. 28 (53), pp. 33-51. Octubre-Diciembre 2019.

Tunja-Boyacá, Colombia. L-ISSN: 0121-1129, e-ISSN: 2357-5328, DOI:

https://doi.org/10.19053/01211129.v28.n53.2019.9745 